\title{
QUEST FOR COSMOS SUBMILLIMETER GALAXY COUNTERPARTS USING CARMA AND VLA: IDENTIFYING THREE HIGH-REDSHIFT STARBURST GALAXIES
}

\author{
V. Smolčić ${ }^{1,2,3,15}$, F. Navarrete ${ }^{2,4}$, M. Aravena ${ }^{5,6}$, O. Ilbert ${ }^{7}$, M. S. Yun ${ }^{8}$, K. Sheth ${ }^{5}$, M. Salvato ${ }^{9}$, H. J. McCracken ${ }^{10}$, \\ C. Diener ${ }^{11}$, I. AretXaga ${ }^{12}$, D. A. Riechers ${ }^{13}$, A. Finoguenov ${ }^{9}$, F. Bertoldi ${ }^{2}$, P. CaPaK ${ }^{13}$, D. Hughes ${ }^{12}$, A. Karim ${ }^{14}$, \\ E. SCHINNERER ${ }^{14}$, N. Z. SCOVILlE ${ }^{13}$, AND G. WILSON ${ }^{12}$ \\ ${ }^{1}$ European Southern Observatory, Karl-Schwarzschild-Strasse 2, D-85748 Garching b. Muenchen, Germany \\ ${ }_{2}$ Argelander Institut for Astronomy, Auf dem Hügel 71, Bonn D-53121, Germany \\ ${ }^{3}$ Physics Department, University of Zagreb, Bijenička cesta 32, 10002 Zagreb, Croatia \\ ${ }^{4}$ Max-Planck Institut für Radioastronomy, Auf dem Hügel 69, D-53121 Bonn, Germany \\ ${ }^{5}$ National Radio Astronomy Observatory, 520 Edgemont Road, Charlottesville, VA 22903, USA \\ ${ }^{6}$ European Southern Observatory, Alonso de Crdoba 3107, Vitacura, Casilla 19001, Santiago 19, Chile \\ ${ }^{7}$ Laboratoire d'Astrophysique de Marseille, Université de Provence, CNRS, BP 8, Traverse du Siphon, F-13376 Marseille Cedex 12, France \\ ${ }^{8}$ Department of Astronomy, University of Massachusetts, Amherst, MA 01003, USA \\ ${ }^{9}$ Max-Planck-Institut für Extraterrestrische Physik, Giessenbachstraße, D-85748 Garching, Germany \\ ${ }^{10}$ Institut d'Astrophysique de Paris, UMR7095 CNRS, Universit Pierre et Marie Curie, 98 bis Boulevard Arago, F-75014 Paris, France \\ ${ }_{11}$ Institute for Astronomy, ETH Zrich, Wolfgang-Pauli-strasse 27, 8093 Zurich, Switzerland \\ 12 Instituto Nacional de Astrofísica, Óptica y Electrónica (INAOE), Aptdo. Postal 51 y 216, 72000 Puebla, Pue., Mexico \\ ${ }^{13}$ Astronomy Department, California Institute of Technology, MC 249-17, 1200 East California Boulevard, Pasadena, CA 91125, USA \\ ${ }^{14}$ Max Planck Institut für Astronomie, Königstuhl 17, Heidelberg D-69117, Germany \\ Received 2011 May 10; accepted 2012 March 21; published 2012 May 4
}

\begin{abstract}
We report on interferometric observations at $1.3 \mathrm{~mm}$ at $2^{\prime \prime}-3^{\prime \prime}$ resolution using the Combined Array for Research in Millimeter-wave Astronomy. We identify multi-wavelength counterparts of three submillimeter galaxies (SMGs; $F_{1 \mathrm{~mm}}>5.5 \mathrm{mJy}$ ) in the COSMOS field, initially detected with MAMBO and AzTEC bolometers at low, $\sim 10^{\prime \prime}-30^{\prime \prime}$, resolution. All three sources-AzTEC/C1, Cosbo-3, and Cosbo-8-are identified to coincide with positions of $20 \mathrm{~cm}$ radio sources. Cosbo-3, however, is not associated with the most likely radio counterpart, closest to the MAMBO source position, but with that farther away from it. This illustrates the need for intermediate-resolution $\left(\sim 2^{\prime \prime}\right)$ mm-observations to identify the correct counterparts of single-dish-detected SMGs. All of our three sources become prominent only at NIR wavelengths, and their mm-to-radio flux based redshifts suggest that they lie at redshifts $z \gtrsim 2$. As a proof of concept, we show that photometric redshifts can be well determined for SMGs, and we find photometric redshifts of $5.6 \pm 1.2,1.9_{-0.5}^{+0.9}$, and $\sim 4$ for AzTEC/C1, Cosbo-3, and Cosbo-8, respectively. Using these we infer that these galaxies have radio-based star formation rates of $\gtrsim 1000 M_{\odot} \mathrm{yr}^{-1}$ and IR luminosities of $\sim 10^{13} L_{\odot}$ consistent with properties of high-redshift SMGs. In summary, our sources reflect a variety of SMG properties in terms of redshift and clustering, consistent with the framework that SMGs are progenitors of $z \sim 2$ and today's passive galaxies.
\end{abstract}

Key words: galaxies: active - galaxies: distances and redshifts - galaxies: high-redshift - galaxies: starburst submillimeter: galaxies

Online-only material: color figures

\section{INTRODUCTION}

Submillimeter galaxies (SMGs) are ultraluminous, dusty starbursting systems with extreme star formation rates (SFRs) in the range of $\sim 100-1000 M_{\odot} \mathrm{yr}^{-1}$ (e.g., Blain et al. 2002). It has been shown that the bulk of this population is between $z \sim 2$ and 3 (e.g., Chapman et al. 2005). But recently a possible high-redshift tail of SMGs has started to emerge (e.g., Younger et al. 2007, 2009; Valiante et al. 2007). To date, only about 10 $z>4$ SMGs have been confirmed (Daddi et al. 2009a, 2009b; Capak et al. 2008, 2011; Schinnerer et al. 2008; Riechers et al. 2010; Smolčić et al. 2011; Coppin et al. 2009, 2010; Knudsen et al. 2010; Cox et al. 2011; Combes et al. 2012). Their number density is still consistent with that expected in cosmological models (Baugh et al. 2005; Coppin et al. 2009; Smolčić et al. 2011). Note however that these $z>4$ studies are not complete, and may even point to the existence of a new or different

\footnotetext{
${ }^{15}$ ESO ALMA COFUND Fellow.
}

SMG population (Wall et al. 2008). The intense starburst that creates the submillimeter bright emission is likely to occur when the bulk of the stellar mass is being assembled in these galaxies; SMGs are generally believed to be the progenitors of today's massive red-and-dead elliptical galaxies which formed in an intense burst at high redshift (e.g., Cimatti et al. 2008). It is therefore critical to study in detail these cosmologically important objects.

SMGs are generally detected in millimeter $(\mathrm{mm})$ and submillimeter (submm) surveys with single-dish telescopes that have large beams $\left(>10^{\prime \prime}\right)$. The next step is then to pinpoint the precise locations of these objects and to match them with their multiwavelength counterparts and obtain a redshift. Finding the real counterpart for an SMG is not trivial because the spatial density of optical/IR galaxies in deep fields is high and usually there are multiple galaxies within one single-dish $\mathrm{mm} /$ submm beam. Deep radio, mid-IR, optical, and UV (and hard X-rays for active galactic nucleus (AGN)) data of higher resolution have been used to identify the right counterpart by tracing the bright 
Table 1

Summary of Observations with the CARMA Interferometer at $230 \mathrm{GHz}$ in D-configuration

\begin{tabular}{|c|c|c|c|c|c|}
\hline Source & $\begin{array}{c}\text { Pointing Position } \\
{[\mathrm{J} 2000]}\end{array}$ & Date & $\begin{array}{l}\text { On-source } \\
\text { Time (hr) }\end{array}$ & $\begin{array}{c}\text { Beam } \\
(\operatorname{arcsec})\end{array}$ & $\begin{array}{c}\mathrm{rms} \\
\left(\mathrm{mJy} \mathrm{beam}^{-1}\right)\end{array}$ \\
\hline $\mathrm{AzTEC} / \mathrm{C} 1$ & $100141.68+022711.80$ & $2009 \mathrm{Mar}$ & 1.5 & $4^{\prime \prime} .2 \times 3^{\prime \prime} .1$ & 2.3 \\
\hline Cosbo-3 & $100057.20+022013.00$ & $2009 \mathrm{Feb}$ & 4.0 & $2^{\prime \prime} .7 \times 1^{\prime \prime} .9$ & 0.7 \\
\hline Cosbo- 8 & $100000.00+020634.00$ & 2009 Mar & 5.4 & $2^{\prime \prime} .6 \times 2 . .4$ & 1.5 \\
\hline
\end{tabular}

star formation (SF) or AGN activity (e.g., Ivison et al. 2007). However, depending on galaxy properties and redshift, these different tracers are likely to introduce identification biases, i.e., provide true identifications for only a fraction of the sample (and likely at low redshifts). In essence, the most efficient and least biased way to associate counterparts is through high-resolution $\mathrm{mm}$ observations. This has to date been a time-consuming process that resulted in a total of $\sim 50$ SMGs detected via mm-interferometry (Downes et al. 1999; Frayer et al. 2000; Dannerbauer et al. 2002; Downes \& Solomon 2003; Genzel et al. 2003; Kneib et al. 2005; Greve et al. 2005; Tacconi et al. 2006; Sheth et al. 2004; Iono et al. 2006; Younger et al. 2007, 2009; Aravena et al. 2010b; Ikarashi et al. 2011; Tamura et al. 2010; Wang et al. 2011; Chen et al. 2011; Neri et al. 2003; Chapman et al. 2008; Hatsukade et al. 2010). To date the largest comprehensive sample of SMGs detected at high resolution via mm-interferometry is that in the COSMOS field (Younger et al. 2007, 2009; Aravena et al. 2010b) and consists of $\sim 20$ sources in total. Here, we present $1.3 \mathrm{~mm}$ imaging at $\sim 2^{\prime \prime}-3^{\prime \prime}$ resolution with the Combined Array for Research in Millimeter-wave Astronomy (CARMA) interferometer of three further SMGs $\left(F_{1 \mathrm{~mm}}>5.5 \mathrm{mJy}\right)$ in the COSMOS field originally detected in the MAMBO- and AzTEC-COSMOS surveys (Bertoldi et al. 2007; Aretxaga et al. 2011). The counterpart association based on previous data was highly ambiguous due to multiple or faint potential radio counterparts lacking optical/NIR detections. We adopt $H_{0}=70$, $\Omega_{M}=0.3, \Omega_{\Lambda}=0.7$.

\section{DATA}

\subsection{COSMOS Survey}

The COSMOS project is a panchromatic (X-ray to radio) survey of an equatorial $2 \mathrm{deg}^{2}$ field. The field has been observed with the major space-based (Chandra-Elvis et al. 2009; Galaxy Evolution Explorer-Zamojski et al. 2007; Hubble Space Telescope-Scoville et al. 2007; Koekemoer et al. 2007; Leauthaud et al. 2007; Spitzer-Sanders et al. 2007; Ilbert et al. 2009; Le Floc' $h$ et al. 2009; Frayer et al. 2009) and ground-based telescopes (Subaru, Canada-France-Hawaii Telescope, UKIRT, NOAO-Capak et al. 2007; Taniguchi et al. 2007; McCracken et al. 2010; Very Large Array (VLA)—Schinnerer et al. 2007, 2010; V. Smolčić et al., in preparation) in more than 30 bands. Here, we additionally use deep UltraVista observations in $Y, J, H, K s$ bands taken between 2009 December and 2010 April (H. J. McCracken et al. 2012, in preparation). Fractions of the field have been surveyed at (sub)mm wavelengths (Bertoldi et al. 2007; Scott et al. 2008; Aretxaga et al. 2011). The three SMGs targeted here were initially detected by MAMBO (Bertoldi et al. 2007) and AzTEC/ASTE (Aretxaga et al. 2011) surveys of the COSMOS field. The deboosted $\sim 1 \mathrm{~mm}$ fluxes are $13.0_{-1.0}^{+1.1} \mathrm{mJy}$ (Aretxaga et al. 2011), $7.45 \pm 1.1 \mathrm{mJy}$, and $5.45 \pm 1.0 \mathrm{mJy}$ (Bertoldi et al. 2007) for AzTEC/C1, Cosbo-3, Cosbo- 8 , respectively (see also Table 2 ).

\subsection{CARMA Observations, Data Reduction, and Source Detection}

We observed the three sources at $1.3 \mathrm{~mm}$ using the CARMA interferometer in a compact-D array-configuration. The targets-AzTEC/C1, Cosbo-3, and Cosbo-8-were observed with 15 antennas (corresponding to 105 baselines) in 2009 February/March for a total on-source time of 1.5, 4.0, and 5.4 hr, respectively. Weather conditions varied between acceptable and very good for $1 \mathrm{~mm}$ observations. The nearby quasar $1058+015$ was observed every 15 minutes for secondary amplitude and phase calibration. The strong calibrator sources 3C 84, 3C 273, and 0854+201 were observed at least once per track for bandpass and flux calibration. Radio pointing was performed at least every $2.5 \mathrm{hr}$ on nearby sources. The resulting total flux calibration is estimated to be accurate within $15 \%-20 \%$.

The upper (lower) sidebands of the $1 \mathrm{~mm}$ receivers were centered at 230 (225) GHz. Each sideband was observed with 45 channels each $31.25 \mathrm{MHz}$ wide, for a total bandwidth of $2.8 \mathrm{GHz}$ $(2 \times 1406.25 \mathrm{MHz})$. For data reduction and analysis, the MIRIAD package was used. The final data cube obtained after flagging (and combination of the data sets from all runs) was collapsed along the frequency axis to obtain $1.3 \mathrm{~mm}$ continuum images. The $u-v$ data were imaged with natural baseline weighting, leading to synthesized clean beam sizes (rms values) of $4^{\prime \prime} .2 \times 3^{\prime \prime} .1\left(2.3 \mathrm{mJy}_{\text {beam }}{ }^{-1}\right), 2^{\prime \prime} .7 \times 1^{\prime \prime} .9\left(0.7 \mathrm{mJy} \mathrm{beam}^{-1}\right)$, and 2 ". $6 \times 2$ 2..4 (1.5 $\left.\mathrm{mJy}_{\text {beam }}{ }^{-1}\right)$ for AzTEC/C1, Cosbo-3, and Cosbo- 8 , respectively. The observations are summarized in Table 1.

The $1.3 \mathrm{~mm}$ CARMA stamps are shown in Figure 1. All three sources are detected at a $\sim 3 \sigma-4 \sigma$ level. We stress that the positions of our $1.3 \mathrm{~mm}$ sources perfectly coincide (within $\left.\lesssim 0^{\prime \prime} 3\right)$ with significant $20 \mathrm{~cm}(1.4 \mathrm{GHz})$ radio detections drawn from the VLA-COSMOS survey (Schinnerer et al. 2007, 2010; see Table 2). As the chance probability of finding a radio source within the CARMA beam (given the radio source number density) is of the order of only $\sim 10^{-4}$, this significantly boosts the validity of our $\mathrm{mm}$ detections. We have extracted the $1.3 \mathrm{~mm}(230 \mathrm{GHz})$ fluxes using the AIPS tasks MAXFIT (that identifies the position with maximum value in a selected pixel array) and JMFIT (that fits a two-dimensional (2D) Gaussian to selected pixel arrays). The flux densities and the corresponding errors are summarized in Table 2. The fluxes are in relatively good agreement (i.e., within $1 \sigma$ ) with those inferred from the MAMBO data, and show a stronger deviation from the AzTEC $1.1 \mathrm{~mm}$ data. This is likely due to a steep spectral index in the rest-frame submm band which translates into a rapid change in flux even within the $1 \mathrm{~mm}$ window. Assuming $\beta=1.0$ and 2.0, we expect a factor between 1.6 and 1.9 discrepancy between the observed $1.3 \mathrm{~mm}$ and $1.1 \mathrm{~mm}$ flux densities.

\section{ANALYSIS AND RESULTS}

\subsection{Multi-wavelength Counterparts and Their Photometry}

Images of the three SMGs at various wavelengths, with $1.3 \mathrm{~mm}$ CARMA contours overlaid, are shown in Figure 1. 


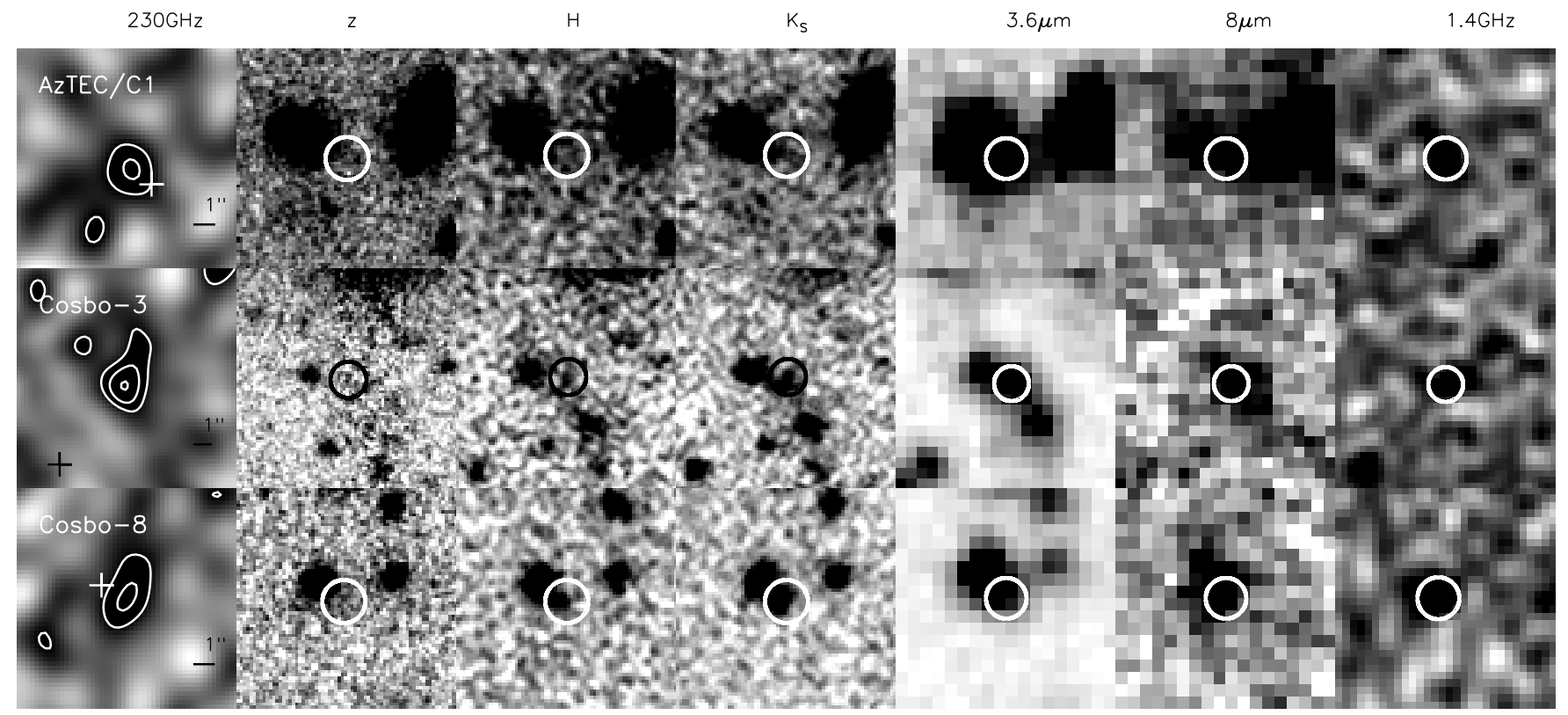

Figure 1. NIR to radio stamps for AzTEC-C1, Cosbo-3, and Cosbo- 8 with $1.3 \mathrm{~mm}$ (CARMA) contours overlaid. The contour levels are at $2 \sigma, 3 \sigma$, and $4 \sigma$ $\left(1 \sigma=2.3,0.7,1.5 \mathrm{mJy}_{\text {beam }}{ }^{-1}\right.$ for AzTEC-C1, Cosbo-3, and Cosbo-8, respectively). The cross indicates the pointing center at $230 \mathrm{GHz}$. The circle ( $2^{\prime \prime}$ in diameter) indicates the CARMA source position.

Table 2

CARMA Detections and VLA Counterparts

\begin{tabular}{lcccccccc}
\hline \hline $\begin{array}{l}\text { Source } \\
\text { Name }\end{array}$ & $\begin{array}{c}\text { CARMA Position } \\
(\mathrm{J} 2000)\end{array}$ & $\begin{array}{c}F_{1.3 \mathrm{~mm}} \\
(\mathrm{mJy})\end{array}$ & $\begin{array}{c}F_{1.2 \mathrm{~mm}} \\
(\mathrm{mJy})\end{array}$ & $\begin{array}{c}F_{1.1 \mathrm{~mm}} \\
(\mathrm{mJy})\end{array}$ & $\begin{array}{c}\text { VLA } \\
\text { Distance }\end{array}$ & $\begin{array}{c}F_{1.4 \mathrm{GHz}} \\
(\mu \mathrm{Jy})\end{array}$ & $\begin{array}{c}\text { Photo- } z \\
(\mathrm{~mm} / \mathrm{radio})\end{array}$ & $\begin{array}{c}\text { Photo- } z \\
\text { UV-MIR }\end{array}$ \\
\hline${\text { AzTEC } / \mathrm{C1}^{\mathrm{a}}}^{\mathrm{a}}$ & $100141.75+022713.06$ & $7.4 \pm 2.3^{\mathrm{b}}$ & - & $13_{-1.0}^{+1.1}$ & $0^{\prime \prime} \cdot 28$ & $44 \pm 10^{\mathrm{c}}$ & $4.3_{-1.4}^{+0.7}$ & $5.6 \pm 1.2$ \\
Cosbo-3 $^{\mathrm{d}}$ & $100056.95+022017.79$ & $5.4 \pm 0.7^{\mathrm{e}}$ & $7.45 \pm 1.1$ & $9.6_{-1.0}^{+1.1}$ & $0^{\prime \prime} \cdot 25$ & $78 \pm 13^{\mathrm{c}}$ & $3.2_{-1.0}^{+0.6}$ & $1.9_{-0.5}^{+0.9}$ \\
Cosbo-8 $^{\mathrm{d}}$ & $095959.92+020633.41$ & $4.8 \pm 1.5^{\mathrm{b}}$ & $5.45 \pm 1.0$ & $3.7_{-1.2}^{+1.1}$ & $0^{\prime \prime} \cdot 35$ & $104 \pm 13^{\mathrm{c}}$ & $1.9_{-0.7}^{+0.5}$ & $\sim 4$ \\
\hline
\end{tabular}

Notes.

a Aretxaga et al. (2011).

${ }^{\mathrm{b}}$ Peak intensity.

c Adopted from the VLA-COSMOS catalogs (Schinnerer et al. 2007, 2010).

${ }^{\mathrm{d}}$ Bertoldi et al. (2007).

${ }^{\mathrm{e}}$ Integral intensity given; the peak intensity is $2.8 \mathrm{mJy}$.

All three sources are coincident (within $\lesssim 00^{\prime \prime} 3$ ) with $20 \mathrm{~cm}$ radio detections as summarized in Table 2 . Only Cosbo- 8 is detected in X-ray emission (within the Chandra-COSMOS survey; Elvis et al. 2009). The multi-wavelength counterparts of our sources become prominent only at NIR wavelengths and are blended by nearby sources. In order to extract the most accurate photometry for these sources, we have carefully deblended the SMG emission (up to the Spitzer/IRAC $8 \mu \mathrm{m}$ band) and extracted its flux as described in the following.

\subsubsection{Calibration of Photometry using the COSMOS Photometric Catalog}

In this section, we describe our photometric extraction procedure that will be applied in the next section to the CARMA-COSMOS UV-MIR counterparts. The photometry of sources in the COSMOS photometric catalog is extracted using aperture techniques, which we also adopt here. To validate our photometric extraction procedure and to estimate its uncertainty, we have drawn $\sim 100$ random sources from the $i$-band-selected catalog (Capak et al. 2007) that also have IR detections and are outside masked regions in the field. Using images at their original resolution (not convolved to a common FWHM; see Capak et al. 2007 for details), we adopt aperture sizes for individual bands as summarized in Table 3. These aperture sizes were cho- sen to achieve the best agreement with the photometry reported in the COSMOS photometric catalog. We also corrected our aperture magnitudes for slight systematic offsets (see Table 3) in order to put them on the COSMOS photometry scale. Thus, our final magnitudes are computed as $m_{\text {apert }}^{\text {final }}=m_{\text {apert }}+m_{\text {offset }}$. Note also that for the IRAC 3.6 and $4.5 \mu \mathrm{m}$ bands we fit the faint magnitude slope separately (as reported in Table 3), and for the Subaru $z^{+}$band we adopt the relation given by McCracken et al. (2010).

We estimate the average error of our magnitudes by statistical propagation of the magnitude errors reported in the catalog and the spread of the (catalog-aperture) magnitude difference (in cases where the average error of the catalog magnitudes is larger than the spread, we adopt the latter as the error of our magnitudes). In summary, our aperture photometry matches that in the COSMOS photometric catalog very well (mean offsets are zero), and the average error of such extracted magnitudes is estimated to be $\sim 0.05$ (see Table 3 for exact values for each band).

\subsubsection{Deblending}

All three sources presented in this work are blended in the near/mid-IR images. We deblend the sources by subtracting 
Table 3

Photometry Calibration

\begin{tabular}{|c|c|c|c|}
\hline Band & $\begin{array}{c}\text { Aperture } \\
\text { Diameter (") }\end{array}$ & $\begin{array}{l}\text { Mag } \\
\text { Offset }\end{array}$ & $\begin{array}{l}\text { Mag } \\
\text { Error }\end{array}$ \\
\hline$g$ & 3 & 0.1123 & 0.034 \\
\hline$r$ & 3 & 0.2514 & 0.016 \\
\hline$i$ & 3 & 0.2619 & 0.034 \\
\hline$z$ & 3 & $0.2203^{\mathrm{a}}$ & 0.039 \\
\hline$J$ & 3 & 0.0828 & 0.063 \\
\hline$H$ & 2.3 & 0.0644 & 0.054 \\
\hline$K_{s}$ & 2 & -0.0390 & 0.080 \\
\hline$Y$-UltraVista & 2 & 0.0000 & 0.040 \\
\hline$J$-UltraVista & 2 & -0.0225 & 0.045 \\
\hline$H$-UltraVista & 2 & -0.1111 & 0.035 \\
\hline$K_{s}$-UltraVista & 2 & -0.1225 & 0.030 \\
\hline $3.6 \mu \mathrm{m}^{\mathrm{b}}$ & 3.8 & $0.0729^{\mathrm{c}}$ & 0.048 \\
\hline $4.5 \mu \mathrm{m}^{\mathrm{b}}$ & 3.8 & $0.0724^{\mathrm{d}}$ & 0.038 \\
\hline $5.8 \mu \mathrm{m}^{\mathrm{b}}$ & 3.8 & 0.0807 & 0.063 \\
\hline $8.0 \mu \mathrm{m}^{\mathrm{b}}$ & 3.8 & 0.0823 & 0.148 \\
\hline IA427 & 2.5 & 0.0828 & 0.052 \\
\hline IA464 & 2.5 & -0.067 & 0.049 \\
\hline IA484 & 2.5 & 0.1418 & 0.067 \\
\hline IA505 & 2.5 & 0.1105 & 0.060 \\
\hline IA 527 & 2.5 & 0.1210 & 0.072 \\
\hline IA574 & 2.5 & -0.0172 & 0.042 \\
\hline IA624 & 2.5 & 0.1786 & 0.051 \\
\hline IA679 & 2.5 & 0.0301 & 0.055 \\
\hline IA709 & 2.5 & 0.0173 & 0.050 \\
\hline IA 738 & 2.5 & 0.1119 & 0.055 \\
\hline IA767 & 2.5 & 0.0263 & 0.087 \\
\hline IA 827 & 2.5 & -0.0875 & 0.036 \\
\hline
\end{tabular}

Notes.

${ }^{\text {a }}$ For the computation of the $z$-band magnitude, we adopt the relation presented in McCracken et al. (2010), and scale it additionally with the offset magnitude given here.

b Total (i.e., aperture-corrected) magnitude.

${ }^{\mathrm{c}}$ In addition, for $m_{3.6 \mu \mathrm{m}}>21.5$ we require $m_{\text {ap }}=1.215 m_{\text {ap }}-5.356$.

${ }^{\mathrm{d}} \mathrm{In}$ addition, for $m_{3.6 \mu \mathrm{m}}>21.5$ we require $m_{\text {ap }}=1.310 m_{\mathrm{ap}}-6.770$.

from the corresponding image a point source centered at the a priori known position of the blending source. The position was obtained either from the $K_{s}$-band image (Cosbo-3 and Cosbo-8) or the pixel with maximum value in the $3.6 \mu \mathrm{m}$ image (AzTEC/C1; see Figures 2-4). The peak value of the point source to be subtracted was optimized by repeating the procedure multiple times until a noise-level residual remained at the position of the blending source. The deblending uncertainty was then obtained from the $1 \sigma$ spread in the extracted magnitudes when varying the peak flux by $\pm 20 \%$. This uncertainty corresponds to $\sim 0.1 \mathrm{mag}$ or better for all bands, and it is then added in quadrature to the photometry error estimated in the previous section in order to obtain the magnitude error.

Assuming a 2D Gaussian point-spread function (PSF) was satisfactory for Cosbo-3 and Cosbo-8 in all affected bands (see Figures 3 and 4), while the source blending AzTEC/C1 appears to have internal structure in IRAC 3.6 and $4.5 \mu \mathrm{m}$ images. Rather than modeling AzTEC/C1 using a more complex (and therefore more uncertain) model, we simply isolate the blending source within an aperture of $2^{\prime \prime}$ radius, mirror it around its diagonal, and then subtract this from the image (see Figure 2). This will obviously cause oversubtraction in the part of the aperture not associated with the source of interest, while it can be assumed that the contribution of the blending source has been well subtracted from the source of interest. The blended
Table 4

Multi-wavelength Photometry

\begin{tabular}{lccc}
\hline \hline Telescope/Band & \multicolumn{3}{c}{ AB Magnitude } \\
\cline { 2 - 4 } & AzTEC/C1 & Cosbo-3 & Cosbo-8 \\
\hline Subaru $/ g^{+}$ & $>26.5$ & $>26.5$ & $>26.5$ \\
Subaru $/ r^{+}$ & $>26.5$ & $>26.5$ & $26.8 \pm 0.3$ \\
Subaru $/ i^{+}$ & $>26.1$ & $>26.1$ & $25.8 \pm 0.3$ \\
Subaru/ $z^{+}$ & $>25.1$ & $>25.1$ & $24.8 \pm 0.3$ \\
UltraVista/J & $>23.9$ & $24.15 \pm 0.19$ & $>23.9$ \\
UltraVista/H & $>22.7$ & $23.64 \pm 0.18$ & $23.44 \pm 0.20$ \\
UltraVista/K & $>22.4$ & $22.80 \pm 0.18$ & $22.50 \pm 0.20$ \\
Spitzer/3.6 $\mu \mathrm{m}$ & $21.12 \pm 0.11$ & $20.78 \pm 0.11$ & $21.20 \pm 0.11$ \\
Spitzer/4.5 $\mu \mathrm{m}$ & $21.28 \pm 0.11$ & $20.48 \pm 0.11$ & $20.81 \pm 0.11$ \\
Spitzer $/ 5.8 \mu \mathrm{m}$ & $20.83 \pm 0.12$ & $20.35 \pm 0.12$ & $20.36 \pm 0.12$ \\
Spitzer $/ 8.0 \mu \mathrm{m}$ & $20.12 \pm 0.18$ & $19.96 \pm 0.18$ & $20.12 \pm 0.18$ \\
JCMT/AzTEC 1.1 mm & $13.62_{-0.08}^{+0.09}$ & $13.94_{-0.11}^{+0.12}$ & $14.98_{-0.35}^{+0.32}$ \\
IRAM 30 m/MAMBO $1.2 \mathrm{~mm}$ & $\ldots$ & $14.22 \pm 0.16$ & $14.56 \pm 0.20$ \\
CARMA 1.3 mm & $14.23 \pm 0.34$ & $14.57 \pm 0.14$ & $14.70 \pm 0.34$ \\
VLA 20 cm & $19.80 \pm 0.25$ & $19.17 \pm 0.19$ & $18.86 \pm 0.14$ \\
\hline
\end{tabular}

Notes. Magnitudes are total magnitudes already corrected for reddening. Limits are either adopted from Capak et al. $\left(2007, g^{+}, r^{+}, i^{+}, z^{+}\right)$or extracted from the aperture flux $\left(J, H, K_{s}\right)$.

and deblended image cutouts for our sources are shown in Figures 2-4, and the extracted photometry for each source is given in Table 4.

\subsection{Photometric Redshifts}

In this section, we first investigate photometric redshift estimates for SMGs based on a sample of eight interferometrically observed SMGs with spectroscopic redshift spanning a broad redshift range of $z \sim 1-5$ present in the COSMOS field (Section 3.2.1). Showing that photometric redshifts can be reliably calculated for SMGs, we then derive photometric redshifts using the same method for the three CARMACOSMOS SMGs analyzed here (Section 3.2.2).

\subsubsection{Calibration of Photometric Redshifts for SMGs}

Photometric redshifts are computed by fitting optimized spectral templates to the spectral energy distribution (SED) of a given galaxy, leaving redshift as a free parameter. The redshift is then determined via a $\chi^{2}$ minimization procedure. We use the Hyper-z code to compute photometric redshifts for our SMGs with the same parameterization as in Wardlow et al. (2010, 2011): extinction assuming a Calzetti et al. (2000) law, with reddening $\left(A_{V}\right)$ varied from 0 to 5 , and an allowed redshift range of 0-7. Based on $~ 30$ SMGs with spectroscopic redshifts, drawn from the LESS survey, Wardlow et al. (2011) have shown that photometric redshifts for SMGs derived with Hyper-z using the above-mentioned parameterization are estimated accurately (they find a median offset between the spectroscopic and photometric redshifts of $0.023 \pm 0.021$; see also their Figure 1).

In general, the quality of photometric redshifts will depend on the choice of the spectral template library to be fit. Therefore, in addition to the templates provided by Hyper-z (similar to those used by Wardlow et al., 6T hereafter; see below), we also test other sets of template libraries. Our spectral model libraries are summarized as follows.

$2 T$. Only two-burst and constant star formation history (SFH) - templates drawn from the Bruzual \& Charlot (2003) library (and provided with Hyper-z). 

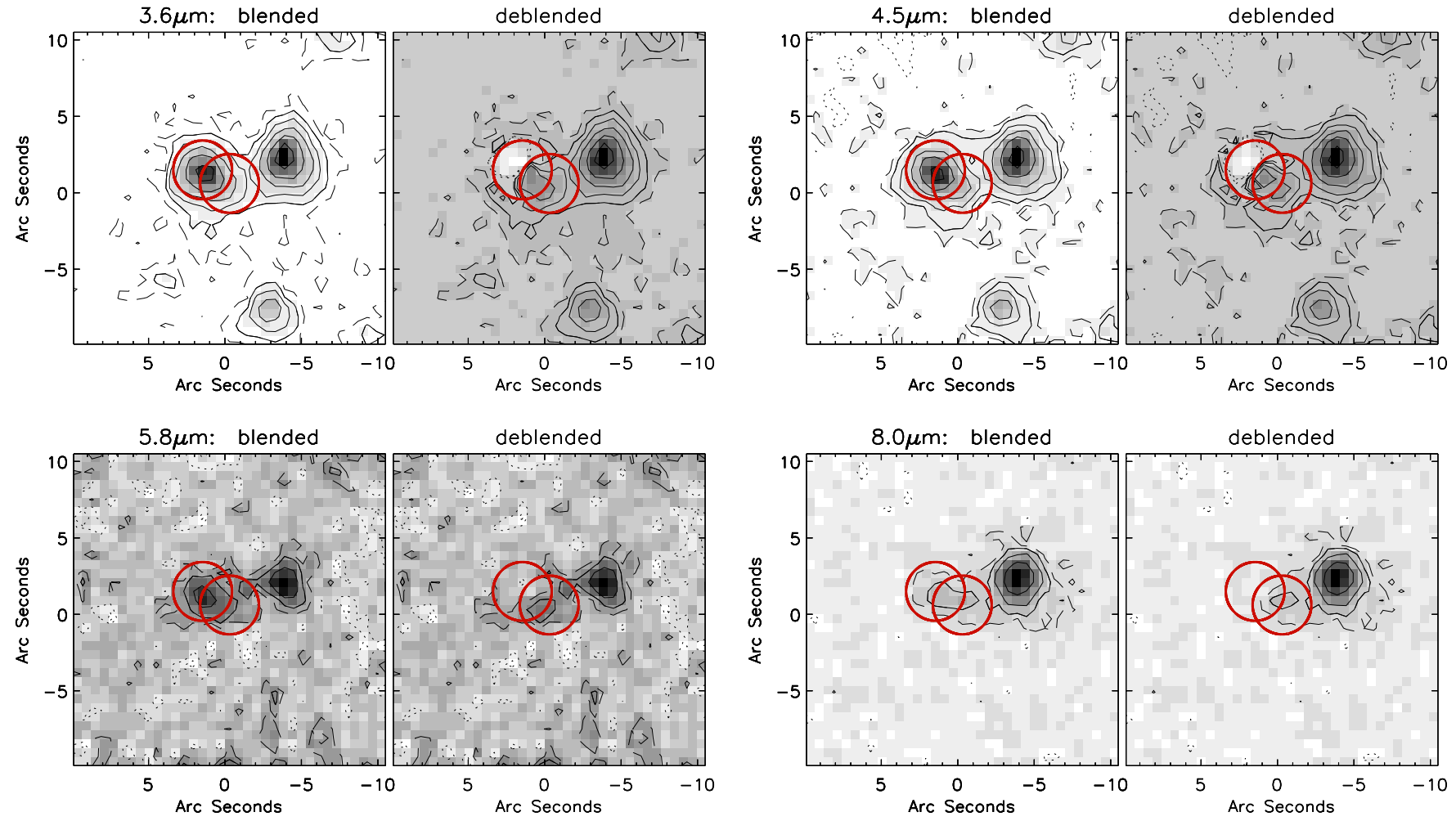

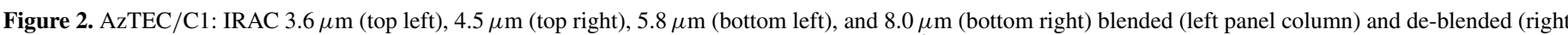

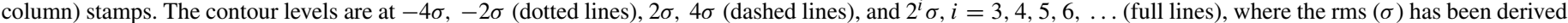

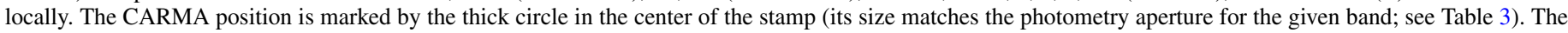

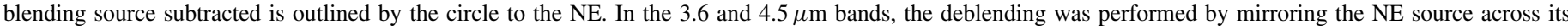

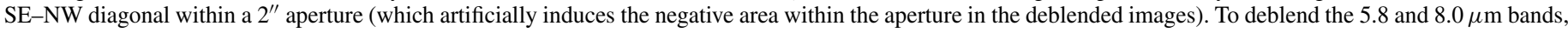
a point source was subtracted.

(A color version of this figure is available in the online journal.)

6T. Six templates provided by the Hyper-z code: burst, four exponentially declining SFHs (SFR $\propto e^{-t / \tau}$, where $t$ is the time, and $\tau=0.3,1,3$, and 5 Gyr) and a constant SFH. This selection of $\mathrm{SFH} /$ templates is similar to the approach used by Ilbert et al. (2009) to compute stellar masses with LePhare.

M. Spectral templates developed in GRASIL (Silva et al. 1998; Iglesias-Páramo et al. 2007) and optimized for SMGs by Michałowski et al. (2010).

For this analysis, we use eight SMGs in the COSMOS field with available spectroscopic redshifts of counterparts determined interferometrically (Capak et al. 2008, 2011; Schinnerer et al. 2008; Riechers et al. 2010; Smolčić et al. 2011; A. Karim et al., in preparation; K. Sheth et al., in preparation). We compute the photometric redshifts for these SMGs as described above and show the total $\chi^{2}$ distribution as a function of redshift in Figure 5. The overall match between the most probable photometric redshift (corresponding to the minimum $\chi^{2}$ ) and the spectroscopic redshift is highly satisfactory. There are no catastrophic outliers. For source AzTEC-3 at $z_{\text {spec }}=5.3$ (Capak et al. 2011; Riechers et al. 2010), there are two $\chi^{2}$ minima. However, the low-redshift peak can be disregarded given that the galaxy is not detected in the radio. If it were a lowredshift SMG, one would expect a strong radio detection given the depth of the VLA-COSMOS survey.

Overall, all templates reach similar solutions, and the best agreement (i.e., tightest $\chi^{2}$ distribution) between the spectroscopic and photometric redshifts is reached when using the Michałowski et al. (2010) spectral templates, and hereafter we
Table 5

Best-fit Properties Given by the Photometric Redshift Computation

\begin{tabular}{lcc}
\hline \hline Source & Template & Hyper-z $A_{V}{ }^{\mathrm{a}}$ \\
\hline AzTEC/C1 & SMMJ030226.17+000624.5 & 0.00 \\
Cosbo-3 & LESSJ033229.4-275619 & 2.55 \\
Cosbo-8 & SMMJ131215.27+423900.9 & 3.00 \\
\hline
\end{tabular}

Notes.

a Reddening computed by Hyper-z; note that the templates already have intrinsic reddening as defined by Michałowski et al. (2010; see their Table A3).

${ }^{\mathrm{b}}$ Best-fit template $/ A_{V}$ for both $\chi^{2}$ minima.

adopt these for our photometric redshift computation. A direct comparison between the photometric (based on $\mathrm{M}$ templates) and spectroscopic redshifts is given in Figure 6. The errors indicate the $99 \%$ confidence interval. We find a median of -0.03 , and a standard deviation of 0.08 in the $\left(z_{\text {phot }}-z_{\text {spec }}\right) /(1+$ $\left.z_{\text {spec }}\right)$ distribution. We conclude that our photometric redshift computation is accurate for SMGs as expected based on results from previous studies (e.g., Daddi et al. 2009b; Wardlow et al. 2010, 2011; Yun et al. 2012).

\subsubsection{Redshifts for CARMA-COSMOS SMGs}

Following the same approach as described in the previous section, we compute the photometric redshifts for AzTEC/C1, Cosbo-3, and Cosbo-8 using their extracted (deblended) photometry (see Table 4). The results are presented in Figure 7 and Table 5. We find photometric redshifts of $5.6 \pm 1.2$ 

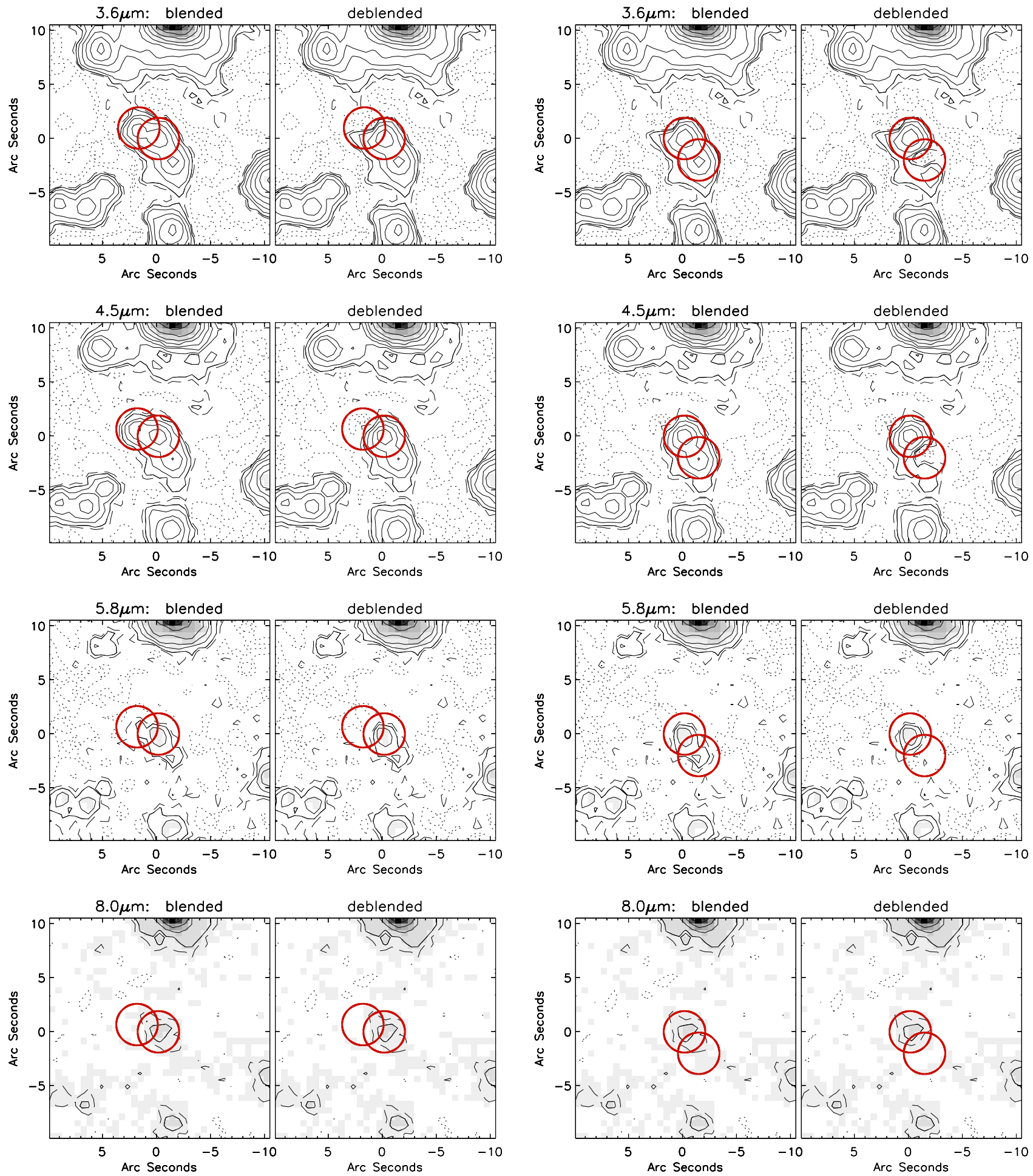

Figure 3. Cosbo-3 (indicated by the thick circle in the center of the stamp) is blended by two (NE and SW) nearby sources (also indicated by circles). From top to bottom, the rows show IRAC 3.6, 4.5, 5.8, and $8.0 \mu \mathrm{m}$ bands, respectively. The contour levels are at $-4 \sigma,-2 \sigma$ (dotted lines), $2 \sigma, 4 \sigma$ (dashed lines), and $2^{i} \sigma, i=3,4,5,6, \ldots$ (full lines), where the rms $(\sigma)$ has been derived locally. The deblending was done by iteratively subtracting a point source (2D Gaussian) in all four IRAC bands.

(A color version of this figure is available in the online journal.)

and $1.9_{-0.5}^{+0.9}$ for AzTEC/C1 and Cosbo-3, respectively. We stress that our photometric redshift for Cosbo-3 is consistent (within $\Delta z=0.5)$ with the recently confirmed spectroscopic redshift (D. A. Riechers et al. 2012, in preparation), affirming both our computation of the UV-MIR-based photometric redshifts, and deblending technique.
Cosbo- 8 is detected in the $\mathrm{X}$-rays within the Chandra-COSMOS survey $\left(F_{0.5-10 \mathrm{keV}}=(2.49 \pm 0.65) \times\right.$ $10^{-15} \mathrm{erg} \mathrm{cm}^{-2} \mathrm{~s}^{-1}$ ) suggesting the presence of an AGN. As AGNs are variable sources with featureless power-law SEDs, special treatment for photometric redshift estimates is required. In the COSMOS survey, Salvato et al. $(2009,2011)$ have 

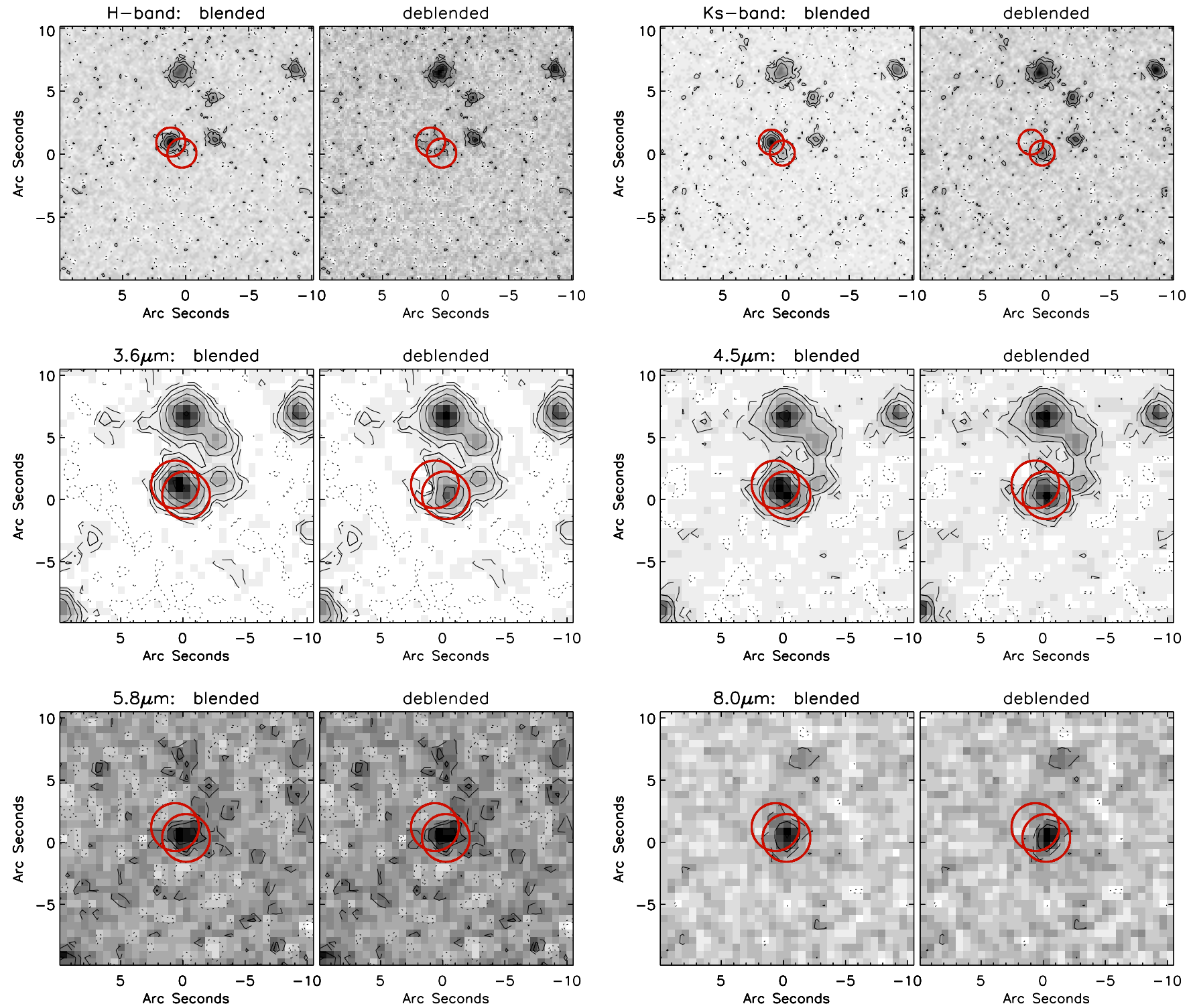

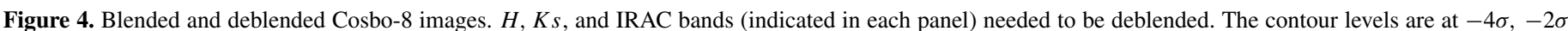

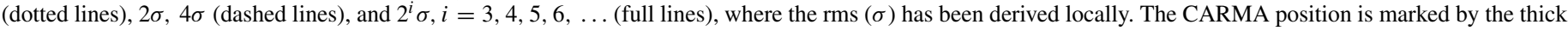

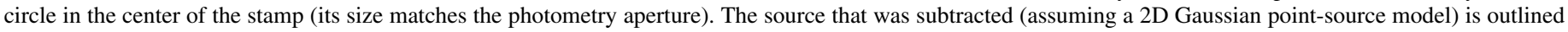
by the circle to the NE.

(A color version of this figure is available in the online journal.)

optimized the photometric redshift computation for X-rayselected sources reaching an accuracy of $\sigma_{\Delta z /\left(1+z_{\text {spec }}\right)} \sim 0.015$. Salvato et al. (2011) find that the Chandra-COSMOS source associated with Cosbo-8 (CID 838; see Civano et al. 2012) has a photometric redshift corresponding to $z_{\text {phot }}=0.82 \pm 0.02$ best fit by a normal galaxy template. However, the photometry is contaminated by the presence of a nearby object. Deblending and extracting the photometry as described here, with the addition of $J, H, K s$ from UltraVista, the photometric redshift value ranges from $z_{\text {phot }}=3.6$ to 4.3 , depending on the luminosity prior adopted (i.e., assuming a luminosity typical of a lowluminosity AGN, i.e., $-8<M_{B}<-24$, or typical of a QSO, i.e., $-20<M_{B}<-30$, respectively; Véron-Cetty \& Véron 2001). Assuming a low-luminosity AGN, the best-fit template is a Seyfert 1.8 drawn from the Polletta et al. (2007) library while assuming a luminosity typical of QSOs, the best-fit template is a hybrid created using an ULIRG (IRAS22491) and a QSO (see Figure 7 and Salvato et al 2009 for details). The solutions are consistent within $1 \sigma$ as in both cases the redshift probability distribution function shows a broad range of possible solutions, rather than a well-defined unique peak. These solutions are also consistent with the photometric redshift value computed as described in the previous section which yields two $\chi^{2}$ minima (at $z \sim 3$ and $z \sim 4$; see Figure 7). Comparing the results from the various spectral libraries, the best $\chi^{2}$ value is obtained when using a QSO prior yielding a redshift of $z_{\text {phot }}=4.1_{-0.5}^{+0.2}$ (where the error is a $1 \sigma$ error; see Figure 7). Hereafter we take $z \sim 4$ as the best redshift estimate for this source, noting that spectroscopic redshift follow-up is required to disentangle between the various photometric redshift solutions for this SMG.

For comparison, the mm-to-radio flux ratios of the sources, regularly utilized as a redshift estimate for SMGs (Carilli \& Yun 1999), suggest that all three sources lie at high $(z \gtrsim 2)$ redshift (see Table 2). An improved version of the dust-independent Carilli \& Yun (1999) redshift estimator via the observed mmto-radio flux density ratio (Yun et al. 2012) yields redshifts 

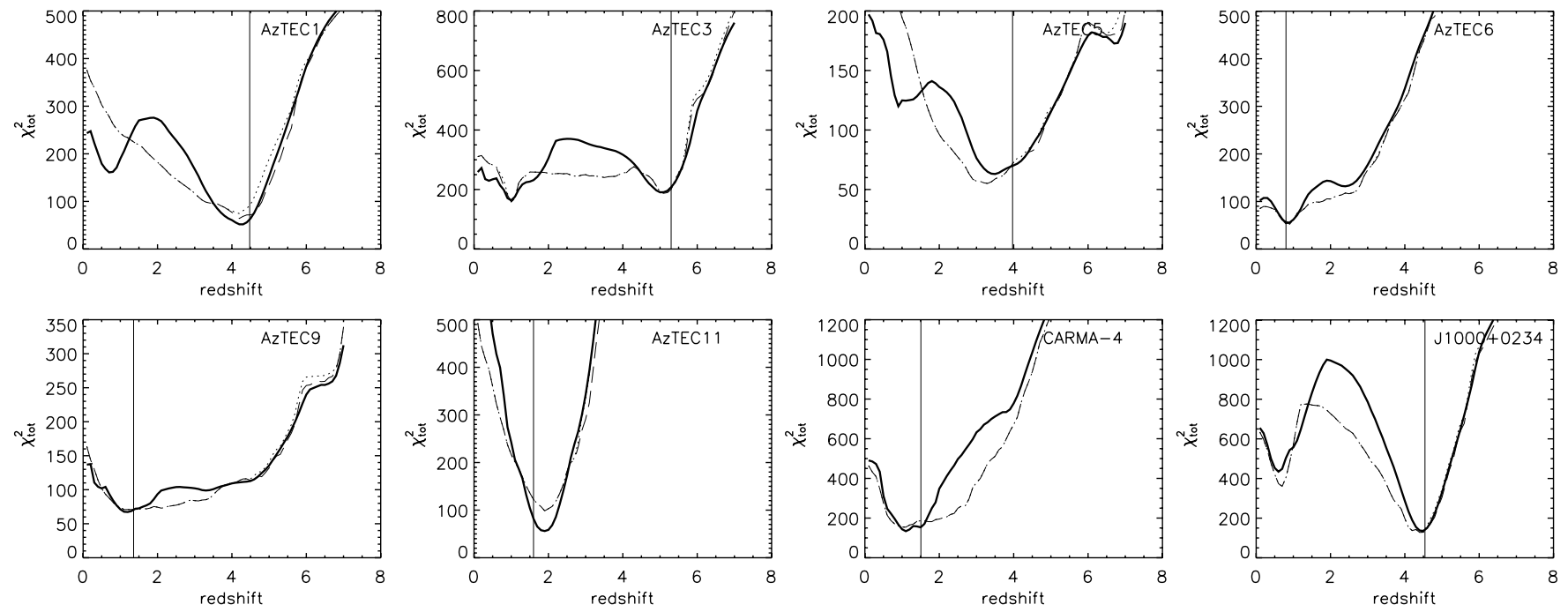

Figure 5. Full $\chi^{2}$ distribution of the photometric redshift determination for SMGs in the COSMOS field with interferometrically determined counterparts with spectroscopic redshifts. The photometric redshifts were derived using various sets of spectral models (see the text for details): $2 \mathrm{~T}$ (dotted lines), 6T (dashed-lines), and M (full lines). The spectroscopic redshifts are indicated by vertical lines. Note that for AzTEC-3 the radio non-detection rules out the first $\chi^{2}$ minimum.

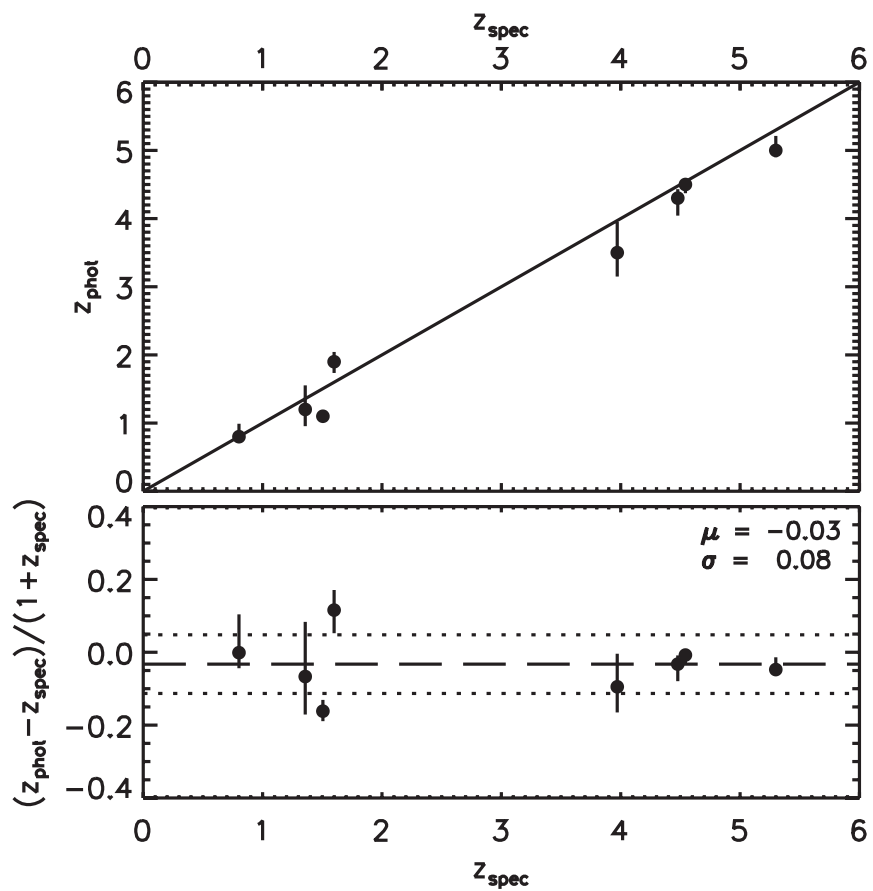

Figure 6. Comparison of spectroscopic and photometric redshifts for eight SMGs from the COSMOS field using the Michałowski et al. (2010) spectral templates. The errors show the $99 \%$ confidence interval. The median offset and standard deviation of the $\Delta z /\left(1+z_{\text {spec }}\right)$ distribution are indicated in the bottom panel.

of $4.3_{-1.4}^{+0.7}, 3.2_{-1.0}^{+0.6}$, and $1.9_{-0.7}^{+0.5}$ for AzTEC/C1, Cosbo-3, and Cosbo-8, respectively (using AzTEC $1.1 \mathrm{~mm}$ measurements for all sources).

In summary, we find photometric redshifts of $5.6 \pm 1.2$, $1.9_{-0.5}^{+0.9}$, and $\sim 4$ for AzTEC/C1, Cosbo-3, and Cosbo-8, respectively. Below we summarize the properties of each SMG.

\subsection{Properties of Individual Sources}

\subsubsection{AzTEC/C1}

AzTEC/C1 has neither a $J-, H$-, or $K s$-band counterpart, while it can be associated with a source at $3.6 \mu \mathrm{m}$ (and becoming most prominent at $8.0 \mu \mathrm{m}$ ) that is, however, strongly blended with a bright source 1".97 to the NE (see Figures 1 and 2). The radio counterpart of AzTEC/C1 is only 0'.28 away from the reported CARMA detection. ${ }^{16}$ The deblended images and photometry for AzTEC/C1 are presented in Figure 2 and Table 4.

The mm-to-radio flux ratio of AzTEC/C1 yields a redshift of $z_{\mathrm{mm} / \text { radio }}=4.3_{-1.4}^{+0.7}$, consistent with the photometric redshift of $z_{\text {phot }}=5.6 \pm 1.2$ derived from its UV-MIR photometry. The inferred high redshift is consistent with the source not being detected at wavelengths shorter than $3 \mu \mathrm{m}$. At such a redshift (4.3-5.6), the radio flux density of the galaxy $\left(F_{20 \mathrm{~cm}}=\right.$ $44 \pm 10 \mu \mathrm{Jy})$ would imply a $20 \mathrm{~cm}$ luminosity of $(6-10) \times$ $10^{24} \mathrm{~W} \mathrm{~Hz}^{-1}$. If the entire radio emission arises from SF in the galaxy, and if at these redshifts locally determined radio-SFR calibrators (Bell 2003; Yun et al. 2001) can be applied (as would be suggested by the constancy of the FIR-radio correlation out to high redshifts; Sargent et al. 2010a, 2010b; Murphy 2009), this radio luminosity would imply an SFR of $\sim 3200-5600 M_{\odot} \mathrm{yr}^{-1}$. This is somewhat in excess of expectations for typical SMGs, thus it may be possible that part of the (radio) emission from this source arises from black hole accretion. However, it is worth noting that such properties are not unusual for $z>4$ SMGs. For example, the properties of AzTEC/C1 are very similar to those of AzTEC-1 - the brightest SMG in the AzTEC/James Clerk Maxwell telescope (JCMT) COSMOS survey (Younger et al. 2007, 2009; Smolčić et al. 2011; $F_{20 \mathrm{~cm}}=42 \mu \mathrm{Jy}, z=4.6$ ).

\subsubsection{Cosbo-3}

A source coincident with the position of Cosbo-3 is detected in the $J$ band, as well as in the longer wavelength bands. In

\footnotetext{
${ }^{16}$ Based on the signal to noise ratio $(\mathrm{S} / \mathrm{N})=4.4$ radio detection at a resolution of $\Theta=1^{\prime \prime} .5$, the expected astrometric accuracy is $\Theta /(\mathrm{S} / \mathrm{N})=0^{\prime \prime} .34$ (note that the overall VLA-COSMOS astrometric accuracy is estimated to be better than 0.'130; see Schinnerer et al. 2007 for details). In order to assess the astrometric accuracy of our CARMA detection, we imaged 3C273, our secondary calibrator $\sim 37 \mathrm{deg}$ away from the AzTEC/C1 field phase center. We recover its position within 0 '. $^{\prime} 11$ of the nominal position. This yields that the positional uncertainty of AzTEC/C1 is likely better than this value, and thus it rules out the possibility that the bright IR galaxy $\sim 2^{\prime \prime}$ away from AzTEC/C1 (which also corresponds to the closest source to AzTEC/C1 detected in the optical) is its counterpart (or that of the radio source).
} 

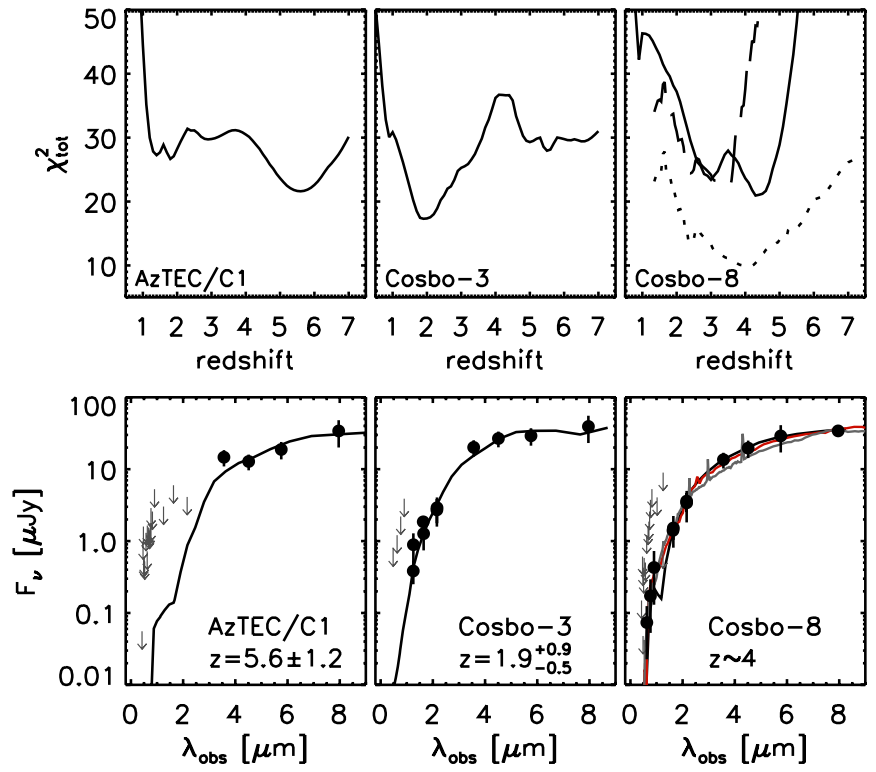

Figure 7. Top panel: total $\chi^{2}$ distribution as a function of redshift for our SMGs (indicated in the panels). Solid curves show the solution based on the Michałowski et al. (2010) spectral model library used to derive the photometric redshifts as described in Section 3.2. For Cosbo-8 (right panel), we also show the total $\chi^{2}$ distribution when using model libraries with AGN templates and a low-luminosity AGN prior (dashed line) and a QSO prior (dotted line) are used (see the text for details). Bottom panel: the spectral energy distribution for our SMGs (indicated in the panels; dots and arrows for $5 \sigma$ upper limits) with the best-fit spectral model (corresponding to that yielding the minimal $\chi^{2}$ value) overplotted (black, gray, and red lines show the best-fit Michałowski et al., AGN, and QSO library spectra, respectively). The best-fit redshift is also indicated.

(A color version of this figure is available in the online journal.)

the Spitzer images, two surrounding sources ( $11^{\prime \prime} 9$ to the NE and 2 ." 4 to the SW, respectively) are blending its IR emission (see Figures 1 and 3). The deblended images and photometry for AzTEC/C1 are presented in Figure 3 and Table 4.

Within the MAMBO 11" beam, there are two radio sources present, at separations of $1^{\prime \prime} .3$ and $5^{\prime \prime} .9$, respectively. Contrary to expectations, the Cosbo- $3 \mathrm{~mm}$-source identified by CARMA is coincident with the NW radio source and not the radio source (at $z_{\text {photo }}=2.4$ ) closest to the mm-source identified by MAMBO (Bertoldi et al. 2007). Although consistent (within $1 \sigma$ ) with the MAMBO $1.1 \mathrm{~mm}$ flux, the CARMA $1.3 \mathrm{~mm}$ flux density is somewhat lower. Thus, it may be possible that Cosbo-3 at $\sim 11^{\prime \prime}$ resolution is itself a blend of two mm-sources, one of which was not detected within the CARMA $1.3 \mathrm{~mm}$ sensitivity. Our CARMA observations put a $3 \sigma$ upper limit to the emission of a potential second mm-source of $2.1 \mathrm{mJy}$ at $1.3 \mathrm{~mm}$.

Cosbo-3 was found to be located in a strong overdensity $(30 \times$ higher than the field) of star-forming galaxies (Aravena et al. 2010a). Thus, it is possible that part of the MAMBO emission is distributed over several sources, consistent with our CARMA observations. All the galaxies in the overdensity have photometric redshifts in the range $z=2.2-2.4$, providing strong statistical support to the photometric redshift of our identified counterpart.

The mm-to-radio flux ratio suggests a redshift of $z=$ $3.2_{-1.0}^{+0.6}$. Our photometric redshift, based on the deblended UV-MIR data, yields $z_{\text {phot }}=1.9_{-0.5}^{+0.9}$, which is consistent with the source's spectroscopic redshift (D. A. Riechers et al. 2012, in preparation) and closer to the photometric redshift of the surrounding overdensity. Assuming the UV-MIR-based photometric redshift value, the source's $20 \mathrm{~cm}$ radio flux density $\left(F_{20 \mathrm{~cm}}=78 \pm 13 \mu \mathrm{Jy}\right)$ implies a radio luminosity of $\sim 2 \times 10^{24} \mathrm{~W} \mathrm{~Hz}^{-1}$ and an SFR of $\sim 900 M_{\odot} \mathrm{yr}^{-1}$. Scaling an Arp 220 template, we find an IR luminosity of $L_{\mathrm{IR}} \sim 1.5 \times 10^{13} L_{\odot}$. The redshift and SFR of Cosbo-3 are fairly typical for SMGs, found to form stars at similar rates and populating the redshift range $z=2-3$ (e.g., Chapman et al. 2005; Wardlow et al. 2010; Yun et al. 2012).

\subsubsection{Cosbo-8}

A source coincident with the position of Cosbo- 8 is detected in the radio band at high significance $\left(F_{20 \mathrm{~cm}}=104 \pm 13 \mu \mathrm{Jy}\right)$. As in the case of the other two SMGs, in Spitzer images it is blended with a source $1^{\prime \prime} .2$ to the NE (see Figures 1 and 4). Its deblended photometry is presented in Table 4 and the deblended images in Figure 4.

The mm-to-radio flux ratio suggests a redshift of $z=1.9_{-0.7}^{+0.5}$. The UV-MIR-based photometric redshift is in the range of $z \sim 3.6-4.3$ (using spectral models typical for AGNs as this source is detected in the X-rays by Chandra). Assuming $z \sim 4$, the radio flux of Cosbo-8 implies a $20 \mathrm{~cm}$ luminosity of $\sim 10^{25} \mathrm{~W} \mathrm{~Hz}^{-1}$ and a radio-based SFR of $\sim 6400 M_{\odot} \mathrm{yr}^{-1}$, an IR luminosity of $L_{\mathrm{IR}} \sim 1.1 \times 10^{13} L_{\odot}$ (based on a scaled Arp 220 template), and an IR-based SFR of $\sim 1700 M_{\odot} \mathrm{yr}^{-1}$ (Bell 2003). The difference in the radio- and IR-based SFRs suggests the presence of an AGN also at radio wavelengths.

Cosbo- 8 corresponds to a point source detected at high significance in both the full C-COSMOS and the best-PSF C-COSMOS data sets with 15 (2.3 estimated background) and 5 (0 background) counts in the $0.5-2 \mathrm{keV}$ band, respectively. Using the count rate-to-flux conversion factors from Puccetti et al. (2009), we obtain a flux of the source in the $0.5-2 \mathrm{keV}$ band of $(3.8 \pm 1.1) \times 10^{-16} \mathrm{erg} \mathrm{s}^{-1} \mathrm{~cm}^{2}$. Assuming $z \sim 4$ and $\Gamma=1.4$, this corresponds to a rest-frame luminosity in the $2-10 \mathrm{keV}$ band of $6.8 \times 10^{43} \mathrm{erg} \mathrm{s}^{-1}$, which corresponds to the level of the emission of a typical AGN (using $\Gamma=2$ results in a $10 \%$ downward revision of the luminosity).

In the MAMBO-COSMOS area to date, only one other SMG (Cosbo-11) has been confirmed as an X-ray-detected AGN (type-1 QSO at $z_{\text {spec }}=1.83$; Aravena et al. 2008). Cosbo-11 is likely ongoing a merger and shows radio and IR luminosities consistent with purely SF activity. Based on the duality of properties showing both properties of QSO and starburst, it has been classified as a starburst-to-QSO "transition" system. Cosbo-8, on the other hand, is also ongoing a major starburst as implied by its IR luminosity, however, it shows an excess of radio emission with respect to that expected from the IR SED. This suggests that the AGN in this case is having a more important role in the bolometric output. Thus, this source could also be classified as a starburst-QSO transition object, but possibly in a more advanced stage when the AGN starts to dominate the SED.

\section{SUMMARY AND DISCUSSION}

In order to unambiguously determine the multi-wavelength counterparts of three $F_{1 \mathrm{~mm}}>5.5 \mathrm{mJy}$ SMGs in the COSMOS field (initially detected with MAMBO and AzTEC bolometers at low, $>10^{\prime \prime}$, resolution), we performed interferometric observations at $1.3 \mathrm{~mm}$ and $\sim 2^{\prime \prime}-3^{\prime \prime}$ resolution using CARMA. The observations yielded $3 \sigma-4 \sigma$ detections coincident with positions of $20 \mathrm{~cm}$ radio sources (VLA-COSMOS survey; Schinnerer et al. 2007, 2010). Although all three sources are 
coincident with radio detections, our observations illustrate the need for high-resolution mm-imaging to determine the correct counterparts of bolometer-identified SMGs. Without highresolution mm observations, the counterpart of Cosbo-3 would have been misclassified as our observations associate this SMG with the radio source (out of two radio sources) within the MAMBO beam that is farther away from the MAMBO source center.

All three sources identified here are blended in the MIR by nearby bright galaxies. We have carefully deblended their photometry, and derived photometric redshifts. We find photometric redshift of $z_{\text {phot }}(\mathrm{AzTEC} / \mathrm{C} 1)=5.6 \pm 1.2, z_{\text {phot }}(\mathrm{Cosbo}-3)=$ $1.9_{-0.5}^{+0.9}$, and $z_{\text {phot }}($ Cosbo -8$) \sim 4$. These are consistent with mm-to-radio flux based estimates for AzTEC/C1 and Cosbo$3\left(4.3_{-1.4}^{+0.7}\right.$ and $3.2_{-1.0}^{+0.6}$, respectively), but inconsistent with that inferred for Cosbo-8 $\left(1.9_{-0.7}^{+0.5}\right)$. This is naturally understood as (part of) the radio flux in Cosbo-8 may arise from the associated AGNs identified via the X-ray Chandra detection. An increased radio flux due to processes not related to SF would lead to an artificial decrease in the mm-to-radio flux based redshift.

Our three SMGs seem to show a relatively large redshift spread, comparable to optically selected SMGs, but with a potential bias toward higher redshifts as all three SMGs have been found to be at $z \gtrsim 2$ (e.g., Chapman et al. 2005). In general, although it has been shown that the SMG population peaks between redshifts 2 and 3 (e.g., Chapman et al. 2005; Wardlow et al. 2011; Yun et al. 2012), their exact redshift distribution (and thus their cosmic evolution) is still rather poorly understood. This is mainly related to statistical counterpart selection biases induced by the large single-dish mm-beams (see, e.g., Yun et al. 2012 for a more detailed discussion). This can be avoided by mm-interferometric imaging at intermediate/high angular resolution of complete samples of SMGs. However, generating such samples has been a very time-consuming, and largely unfeasible process, and assembling complete samples of SMG counterparts (and their redshifts) will require surveys with facilities such as ALMA and the Large Millimeter Telescope. Nonetheless, existing $\mathrm{mm}$-interferometric observations of SMGs already suggest that a fraction of these sources (at least at the bright end) is unexpectedly at redshifts $z \gtrsim 4$ (e.g., 4/17 AzTEC/JCMT SMGs detected by SMA/VLA are spectroscopically confirmed to be at $z \gtrsim 4$; Scott et al. 2008; Younger et al 2007, 2009; Capak et al. 2008, 2011; Schinnerer et al. 2008; Riechers et al. 2010; A. Karim et al. 2012, in preparation). In this cosmic epoch to date, there are only about 10 SMGs confirmed (Daddi et al. 2009a, 2009b; Capak et al. 2008, 2011; Schinnerer et al. 2008; Riechers et al. 2010; Smolčić et al. 2011; Coppin et al. 2009, 2010; Knudsen et al. 2010). Given their large SFRs, these very high redshift SMGs are considered to be optimal candidates for the progenitors of $z \sim 2$ massive red galaxies (Cimatti et al. 2008). However, as this population is just starting to emerge, their role in galaxy evolution is still largely unexplored, and efforts to identify such sources and characterize their properties is critical. Based on mm-/radio-interferometry, we have associated AzTEC/C1, the brightest SMG in the AzTEC/ASTE COSMOS survey, with a $\mathrm{MIR} /$ radio source at $z \gtrsim 4$ SMG. Interestingly, its high-redshift, $\mathrm{mm}$ and radio fluxes are comparable to that of AzTEC-1-the brightest SMG in the AzTEC/JCMT COSMOS survey (Scott et al. 2008)—with properties resembling those expected for the progenitors of compact massive red galaxies at $z \sim 2$ (Smolčić et al. 2011).
Studies of SMGs, and the formation of passive red galaxies (often found in the most massive galaxy clusters) suggest that the first may be progenitors of the second (e.g., Michałowski et al. 2010; Hickox et al. 2012). Consistent with this picture, in which strong clustering of SMGs is expected, spatial clustering analysis of $z \lesssim 3$ SMGs find that SMGs cluster strongly (e.g., Hickox et al. 2012), and that they can be statistically associated with galaxy overdensities (Aravena et al. 2010a). Furthermore, only recently have two $z>4$ protoclusters hosting SMGs been identified (Daddi et al. 2009b; Capak et al. 2011), providing valuable laboratories to study structure formation at the earliest cosmic times. Based on $B z K$-selected galaxies, Aravena et al. (2010a) identified a significant galaxy overdensity at $z \sim 2.3$ in the area around Cosbo-3. The $\mathrm{mm} /$ radio-interferometric observations and photometric redshift computation, presented here, strengthen the case that Cosbo- 3 is indeed associated with this galaxy overdensity (rather than being a fore-/background galaxy). This system therefore proves interesting for further studies of the dense environment of SMGs at the peak epoch of this population, linking the properties of $z>4$ protoclusters hosting SMGs and local galaxy clusters.

In summary, we have identified the counterparts of three single-dish-detected SMGs in the COSMOS field via mminterferometry, and presented their (deblended) UV-MIR photometry, and redshift estimates. Such studies are an important step toward reaching unbiased statistical samples of SMGs with accurately determined counterparts and redshifts-a necessary but yet unaccomplished prerequisite for comprehensive studies of physical properties of SMG population(s) and their role in galaxy formation and evolution.

The authors thank the anonymous referee for comments that significantly improved the manuscript. The research leading to these results has received funding from the European Union's Seventh Framework programme under grant agreement 229517. M.S. acknowledges support by the German Deutsche Forschungsgemeinschaft, DFG Leibniz Prize (FKZ HA 1850/28-1). A.K. acknowledges support by the DFG grant SCHI 536/3-3 as part of the Priority Programme 1177 (Witnesses of Cosmic History: Formation and Evolution of Black Holes, Galaxies and their Environment). Support for CARMA construction was derived from the Gordon and Betty Moore Foundation, the Kenneth T. and Eileen L. Norris Foundation, the James S. McDonnell Foundation, the Associates of the California Institute of Technology, the University of Chicago, the states of California, Illinois, and Maryland, and the National Science Foundation. Ongoing CARMA development and operations are supported by the National Science Foundation under a cooperative agreement, and by the CARMA partner universities.

\section{REFERENCES}

Aravena, M., Bertoldi, F., Carilli, C., et al. 2010a, ApJ, 708, L36

Aravena, M., Bertoldi, F., Schinnerer, E., et al. 2008, A\&A, 491, 173

Aravena, M., Younger, J. D., Fazio, G. G., et al. 2010b, ApJ, 719, L15

Aretxaga, I., Wilson, G. W., Aguilar, E., et al. 2011, MNRAS, 415, 3831

Baugh, C. M., Lacey, C. G., Frenk, C. S., et al. 2005, MNRAS, 356, 1191

Bell, E. F. 2003, ApJ, 586, 794

Bertoldi, F., Carilli, C., Aravena, M., et al. 2007, ApJS, 172, 132

Blain, A. W., Smail, I., Ivison, R. J., Kneib, J.-P., \& Frayer, D. T. 2002, Phys. Rep., 369, 111

Bruzual, G., \& Charlot, S. 2003, MNRAS, 344, 1000

Calzetti, D., Armus, L., Bohlin, R. C., et al. 2000, ApJ, 533, 682

Capak, P., Aussel, H., Ajiki, M., et al. 2007, ApJS, 172, 99

Capak, P., Carilli, C. L., Lee, N., et al. 2008, ApJ, 681, L53

Capak, P., Riechers, D., Scoville, N. Z., et al. 2011, Nature, 470, 233 
Carilli, C. L., \& Yun, M. S. 1999, ApJ, 513, L13

Chapman, S. C., Blain, A. W., Smail, I., \& Ivison, R. J. 2005, ApJ, 622, 772

Chapman, S. C., Neri, R., Bertoldi, F., et al. 2008, ApJ, 689, 889

Chen, C.-C., Cowie, L. L., Wang, W.-H., Barger, A. J., \& Williams, J. P. 2011, ApJ, 733, 64

Cimatti, A., Cassata, P., Pozzetti, L., et al. 2008, A\&A, 482, 21

Civano, F., et al. 2012, submitted

Combes, F., Rex, M., Rawle, T. D., et al. 2012, A\&A, 538, L4

Coppin, K. E. K., Chapman, S. C., Smail, I., et al. 2010, MNRAS, 407, L103

Coppin, K. E. K., Smail, I., Alexander, D. M., et al. 2009, MNRAS, 395, 1905

Cox, P., Krips, M., Neri, R., et al. 2011, ApJ, 740, 63

Daddi, E., Dannerbauer, H., Krips, M., et al. 2009a, ApJ, 695, L176

Daddi, E., Dannerbauer, H., Stern, D., et al. 2009b, ApJ, 694, 1517

Dannerbauer, H., Lehnert, M. D., Lutz, D., et al. 2002, ApJ, 573, 473

Downes, D., Neri, R., Greve, A., et al. 1999, A\&A, 347, 809

Downes, D., \& Solomon, P. M. 2003, ApJ, 582, 37

Elvis, M., Civano, F., Vignali, C., et al. 2009, ApJS, 184, 158

Frayer, D. T., Sanders, D. B., Surace, J. A., et al. 2009, AJ, 138, 1261

Frayer, D. T., Smail, I., Ivison, R. J., \& Scoville, N. Z. 2000, AJ, 120, 1668

Genzel, R., Baker, A. J., Tacconi, L. J., et al. 2003, ApJ, 584, 633

Greve, T. R., Bertoldi, F., Smail, I., et al. 2005, MNRAS, 359, 1165

Hatsukade, B., Iono, D., Akiyama, T., et al. 2010, ApJ, 711, 974

Hickox, R. C., Wardlow, J. L., Smail, I., et al. 2012, MNRAS, 421, 284

Iglesias-Páramo, J., Buat, V., Hernández-Fernández, J., et al. 2007, ApJ, 670, 279

Ikarashi, S., Kohno, K., Aguirre, J. E., et al. 2011, MNRAS, 415, 3081

Ilbert, O., Capak, P., Salvato, M., et al. 2009, ApJ, 690, 1236

Iono, D., Peck, A. B., Pope, A., et al. 2006, ApJ, 640, L1

Ivison, R. J., Greve, T. R., Dunlop, J. S., et al. 2007, MNRAS, 380, 199

Kneib, J.-P., Neri, R., Smail, I., et al. 2005, A\&A, 434, 819

Knudsen, K. K., Kneib, J.-P., Richard, J., Petitpas, G., \& Egami, E. 2010, ApJ, 709,21

Koekemoer, A. M., Aussel, H., Calzetti, D., et al. 2007, ApJS, 172, 196

Leauthaud, A., Massey, R., Kneib, J.-P., et al. 2007, ApJS, 172, 219

Le Floc'h, E., Aussel, H., Ilbert, O., et al. 2009, ApJ, 703, 222
McCracken, H. J., Capak, P., Salvato, M., et al. 2010, ApJ, 708, 202

Michałowski, M., Hjorth, J., \& Watson, D. 2010, A\&A, 514, A67

Murphy, E. J. 2009, ApJ, 706, 482

Neri, R., Genzel, R., Ivison, R. J., et al. 2003, ApJ, 597, L113

Polletta, M., Tajer, M., Maraschi, L., et al. 2007, ApJ, 663, 81

Puccetti, S., Vignali, C., Cappelluti, N., et al. 2009, ApJS, 185, 586

Riechers, D. A., Capak, P. L., Carilli, C. L., et al. 2010, ApJ, 720, L131

Salvato, M., Hasinger, G., Ilbert, O., et al. 2009, ApJ, 690, 1250

Salvato, M., Ilbert, O., Hasinger, G., et al. 2011, ApJ, 742, 61

Sanders, D. B., Salvato, M., Aussel, H., et al. 2007, ApJS, 172, 86

Sargent, M. T., Schinnerer, E., Murphy, E., et al. 2010a, ApJ, 714, L190

Sargent, M. T., Schinnerer, E., Murphy, E., et al. 2010b, ApJS, 186, 341

Schinnerer, E., Carilli, C. L., Capak, P., et al. 2008, ApJ, 689, L5

Schinnerer, E., Sargent, M. T., Bondi, M., et al. 2010, ApJS, 188, 384

Schinnerer, E., Smolčić, V., Carilli, C. L., et al. 2007, ApJS, 172, 46

Scott, K. S., Austermann, J. E., Perera, T. A., et al. 2008, MNRAS, 385, 2225

Scoville, N., Aussel, H., Brusa, M., et al. 2007, ApJS, 172, 1

Sheth, K., Blain, A. W., Kneib, J.-P., et al. 2004, ApJ, 614, L5

Silva, L., Granato, G. L., Bressan, A., \& Danese, L. 1998, ApJ, 509, 103

Smolčić, V., Capak, P., Ilbert, O., et al. 2011, ApJ, 731, L27

Tacconi, L. J., Neri, R., Chapman, S. C., et al. 2006, ApJ, 640, 228

Tamura, Y., Iono, D., Wilner, D. J., et al. 2010, ApJ, 724, 1270

Taniguchi, Y., Scoville, N., Murayama, T., et al. 2007, ApJS, 172, 9

Valiante, E., Lutz, D., Sturm, E., et al. 2007, ApJ, 660, 1060

Véron-Cetty, M.-P., \& Véron, P. 2001, A\&A, 374, 92

Wall, J. V., Pope, A., \& Scott, D. 2008, MNRAS, 383, 435

Wang, W.-H., Cowie, L. L., Barger, A. J., \& Williams, J. P. 2011, ApJ, 726, L18

Wardlow, J. L., Smail, I., Coppin, K. E. K., et al. 2011, MNRAS, 415, 1479

Wardlow, J. L., Smail, I., Wilson, G. W., et al. 2010, MNRAS, 401, 2299

Younger, J. D., Fazio, G. G., Huang, J.-S., et al. 2007, ApJ, 671, 1531

Younger, J. D., Fazio, G. G., Huang, J.-S., et al. 2009, ApJ, 704, 803

Yun, M. S., Reddy, N. A., \& Condon, J. J. 2001, ApJ, 554, 803

Yun, M. S., Scott, K. S., Guo, Y., et al. 2012, MNRAS, 420, 957

Zamojski, M. A., Schiminovich, D., Rich, R. M., et al. 2007, ApJS, 172, 468 